ÉGYPTE monde arabe

\section{Égypte/Monde arabe}

30-31 | 1997

Les visions de l'Occident dans le monde arabe

\title{
Gérer les normes extérieures
}

Le penchant occidental de la bienfaisance islamique en Égypte

\section{Patrick Haenni}

\section{OpenEdition}

Journals

Édition électronique

URL : https://journals.openedition.org/ema/1654

DOI : $10.4000 /$ ema. 1654

ISSN : 2090-7273

Éditeur

CEDEJ - Centre d'études et de documentation économiques juridiques et sociales

Édition imprimée

Date de publication : 30 septembre 1997

Pagination : 275-291

ISSN : 1110-5097

Référence électronique

Patrick Haenni, « Gérer les normes extérieures », Égypte/Monde arabe [En ligne], 30-31 | 1997, mis en ligne le 08 juillet 2008, consulté le 07 juillet 2022. URL : http://journals.openedition.org/ema/1654 ; DOI : https://doi.org/10.4000/ema.1654

Ce document a été généré automatiquement le 7 juillet 2022.

Tous droits réservés 


\title{
Gérer les normes extérieures
}

\author{
Le penchant occidental de la bienfaisance islamique en Égypte
}

\section{Patrick Haenni}
« Un prince était vexé de ne s'être employé jamais qu'à la perfection des générosités vulgaires. »
Arthur Rimbaud, « Contes », Illuminations

1 Dans le contexte actuel « [d']aggiornamento des catégories islamiques mobilisées pour rejeter toute occidentalisation de la pensée et toute contamination des valeurs héritées par les modèles occidentaux » (Roussillon, 1995, p. 15), affirmation du religieux et rejet de l'Occident semblent comme les deux faces d'une même médaille. Cette connivence n'est toutefois qu'un des scénarios possibles d'une réalité riche en nuances. D'autres constructions du sens que l'instrumentalisation polémique se profilent, où l'on voit les catégories de la morale musulmane et les schèmes culturels issus de la modernité occidentale tisser des convergences inattendues. Il en va notamment ainsi des conduites de charité1. Celles-ci, bien que systématiquement rapportées aux catégories de l'éthique musulmane, dont on connaît la profondeur historique et la charge identitaire, allaient désormais évoluer en relation avec le reste du monde. Au tournant de ce siècle, le réformiste égyptien M. 'Umar ne prônait pas autre chose lorsqu'il invitait les Égyptiens nantis à « imiter les Occidentaux dans cette pratique des œuvres de bienfaisance auxquels ceux-ci consacrent une grande partie de leur énergie » (1902, p.54). Difficile à affirmer ouvertement aujourd'hui, cette orientation subsistera pourtant au creux de la vague de la réislamisation. La bienfaisance se présente ainsi à l'observateur comme le livre ouvert des rapports à l'Occident où l'on verra le domaine des conduites de charité tour à tour investi par les réformistes de la Nahda, les militants islamistes, le corps religieux proche du pouvoir et enfin les élites économiques actuelles.

2 Cet investissement comprend trois manières de faire successives: "l'encodage " (incorporation de catégories allogènes par leur mise en forme locale), la réaction (repli sur un Soi mythique, un Soi que la pratique de la charité va servir à exprimer), l'appropriation (adoption directe et non polémique des schèmes culturels de l'Autre). 
En somme, bien que constamment sollicités par les appels identitaires ${ }^{2}$, les acteurs de la charité font preuve d'une capacité réelle à se déprendre du cadre religieux qui est censé en régir le sens. Nous suivrons ici les grandes étapes de ces transformations. Après un bref pointage des significations historiques de la charité, l'article s'intéressera à l'utopie réformiste de Rifâ'a al-Tahtâwî (1801-1873), ce réformiste égyptien de la première génération qui chercha à moderniser les conceptions endogènes de la charité, en s'inspirant autant des catégories de l'économie politique occidentale, que des institutions et concepts de la morale religieuse. Nous évoquerons brièvement l'inscription de cette charité dans le projet islamiste avant de nous pencher plus en détail sur le processus de sa délocalisation lorsque, reprise par les entrepreneurs cairotes au cours des années quatre-vingt et quatre-vingt-dix, la charité islamique allait virer à l'entreprise philanthropique, que Haynes (1987, p. 1)) définit comme « a form of gifting that originates principally in Western cultures, where it suggests giving animated by a desire to promote human Welfare».

\section{Les répertoires traditionnels de la bienfaisance ${ }^{3}$}

3 Avant les grandes transformations politiques et économiques du XIX ${ }^{\mathrm{e}}$ siècle, générosité (karâma) rimait avec piété et ancrage local : les œuvres de bienfaisance (a'mâl khayriyya), conformément aux injonctions du texte coranique, étaient destinées en premier lieu aux proches (aqârib). Nommément, et par degré d'importance: la famille nucléaire (usra) puis étendue ('â'ila), les voisins et éventuellement le quartier ou le village. Elles ne dépassaient pas l'horizon étroit de l'espace local ('asabiyya) ${ }^{4}$. Leurs motivations étaient variées : d'abord, le souci de s'acquitter d'un des piliers de la foi, la zakât. La charité avait ensuite une dimension mondaine indéniable, opérant comme marque de prestige et de prééminence sociale. Le droit des pauvres à la richesse des riches, affirmé dans le texte coranique, est devenu dans la pratique sociale un devoir de solidarité des classes fortunées à l'égard des pauvres, devoir dont ces dernières s'acquittaient d'autant plus prestement que leurs largesses avaient pour contrepartie une double accumulation de capital symbolique (prestige, sentiment d'excellence) et social (création de relations d'allégeance et de clientèle).

4 Quoiqu'il en soit, cet ethos de la générosité, investi à la fois de fonctions mondaines et religieuses, restait confiné au cercle communautaire et se désintéressait autant du politique que des réalités extérieures. Cette charité constituait d'abord une observance routinière, un habitus religieux qui se situait en aval du sacré : il s'agissait moins de réfléchir sur la catégorie religieuse et morale de la charité que de se conformer à la perception dominante de ce qu'elle doit être. C'est assurément encore la forme de charité la plus répandue aujourd'hui. Toutefois, dès le XIX siècle, cette orientation "traditionnelle» se double d'une orientation réformiste, comprise comme "effort mené par ceux qui sont intervenus dans le champ social pour l'adapter aux exigences d'une société en mouvement en dehors des grands mouvements politiques » (Ilbert, 1995, p. 371). C'est cette démarche qui retiendra notre attention, car les nouvelles formes de charité auxquelles elle aboutit sont pensées en termes politiques. Et par delà, elles sont immanquablement déterminées par un positionnement vis-à-vis de l'Occident. Car ses auteurs, par la nature même de leur ambition réformiste vont être amenés à chercher en Occident les références nécessaires à l'application de leur modèle. Ce qui se fera deux fois de façon positive, une fois de façon négative. La 
réforme des conduites de charité se réalisera dans trois "programmes", chacun porteur d'une vision spécifique de l'Occident : l'évergétisme (Veyne, 1976) des notables de la fin du XIX ${ }^{e}$ siècle, les «stratégies de bienfaisance" (Jaffrelot, 1994) des mouvements islamiques, et les investissements solidaires des entrepreneurs contemporains.

\section{La charité dans le moule de la réforme}

$5 \quad$ C'est à la fin du XIX siècle que l'exercice de la charité va se projeter dans le champ politique et idéologique. Cette mutation a été favorisée par un encadrement étatique d'un corps social particulièrement lâche qui allait libérer les actions individuelles et la gestion « civile » des espaces locaux. Dans le même temps, émerge une classe d'affaire et de négociants qui a besoin d'ordre et de progrès que les institutions centrales, conséquence du règne britannique indirect, n'assuraient pas, ou assuraient mal. Les notables vont fort naturellement - il en allait de leur intérêt - se mobiliser pour conduire l'effort de réforme sociale, et réactiver pour ce faire les institutions les plus directement opératoires et qui se trouvaient être en l'occurrence les institutions religieuses : les biens de mainmorte (awqâf, pl. de waqf) et les œuvres de bienfaisance (a'mâl khayriyya). Ne raisonnant plus en termes d'obligation religieuse mais de responsabilité sociale, ils appréhendaient la charité non dans ses aspects moraux, mais dans ses implications concrètes. Autant qu'un acte de foi, elle était un outil de leur ambition réformiste.

Dans cette conjoncture, c'est Rifâ'a al-Tahtâwî qui, le premier dans ses Manâhij (Méthodes), va inscrire la notion de charité dans une vision réformiste. Convaincu que ce devoir religieux pouvait être un mécanisme performant de régulation sociale, «il suggérait de mobiliser les anciennes institutions de l'islam (aqwâf) au profit d'interventions nouvelles que l'État ne pouvait prendre en charge [...] C'était passer de la charité de proximité à la philanthropie sociale. Et en venir à une théorie des autorités sociales proches de celles d'un Le Play mais construite sur une argumentation endogène " (Alleaume, 1995, p. 415). Il aspirait à un modèle de société apte à la construction d'une modernité sociale et économique affermie par les apports de l'Occident et prônant le recours aux idiomes religieux pour autant qu'un sens nouveau leur soit conféré : c'était là la visée de la Nahda, le mouvement réformiste. Elle trouvera en Occident plus d'un appui : dans la plus pure tradition saint-simonienne qui tient la religion "pour le meilleur moyen de mobiliser les forces sociales" (Alleaume, à paraître), Rifâ'a al-Tahtâwî développe son projet autour de deux axes. Le « contrôle des mœurs par les préceptes de la morale religieuse » d'abord ; "les intérêts sociaux " ensuite, terme désignant toutes les actions d'édilité et de bienfaisance. C'est cette dualité des objectifs qui le pousse à prôner « la nécessaire collaboration des autorités morales et des industriels» (Alleaume, à paraître) pour assurer au mieux l'encadrement social.

7 C'est en expert que Tahtâwî se saisit de la notion de charité, l'inscrivant dans un projet qui fait l'économie d'une perception idéologique de l'Occident: le rapport à lui n'était pas conçu en termes culturels mais pragmatiques; Tahtâwî recherchait "l'Occident utile », celui qui était susceptible de servir son projet. Il s'inscrivait néanmoins dans le cadre de pensée de la Nahda, laquelle rejetait l'idée d'adoption inconditionnelle des modèles occidentaux. Il appréhendait ainsi l'Occident à partir d'une "position 
identitariste ", qui supposait que l'incorporation de ses schèmes culturels soit effectuée par l'intercession de catégories locales. Il s'exposait par là à la détermination propre de son système de référence: la charité pouvait être légitimement libérée du cadre territorial particulariste traditionnel de la 'asabiyya, mais elle ne pouvait être fondamentalement émancipée du corpus religieux et des cadres institutionnels ${ }^{5}$ qui lui sont liés, condition sine qua non, pourtant, pour que puisse se réaliser l'ambition de Tahtâwî. D'une manière ou d'une autre, son projet supposait en effet que soit minimisé le contrôle exercé par un personnel religieux peu enclin à assumer le redéploiement qu'il préconisait et à prendre en charge des opérations que l'État ne pouvait assurer (aide aux chômeurs, aux négociants en faillite, lutte contre l'usure, etc.). L'absence de réalisations concrètes susceptibles de nourrir l'effort de synthèse réclamé par Tahtâwî sur le terrain intellectuel l'amènera à suspendre sa volonté d'accommoder des conceptions occidentales au corpus endogène de la charité.

Quels enseignements tirer de ces exemples? Principalement que les facteurs favorisant ou entravant la fusion entre deux corpus culturels sont moins à rechercher dans leur contenu que dans l'environnement politique et idéologique dans lequel se réalise leur rencontre. Ce sont en définitive les perceptions des acteurs susceptibles de réaliser les transferts qui déterminent la compatibilité des schèmes culturels locaux avec le "monde» de l'Autre, et non des affinités essentielles que porteraient en eux les éléments de ces corpus, préalablement à leur rencontre.

\section{La charité comme altérité}

9 Le socialisme nassérien, au nom des idéaux égalitaires qu'il se proposait de réaliser, jeta l'opprobre sur l'expression ouverte des richesses privées. Idéologiquement dévalorisées, les conduites de charité étaient de plus risquées car elles visibilisaient les prestataires, exposant leurs biens à la confiscation. L'ambition étatique du régime comme le renforcement silencieux des mouvements religieux qui lui étaient opposés allaient paradoxalement se rejoindre pour occuper l'espace des pratiques de charité, avec des idéologies nouvelles, différentes dans leur contenu, mais partageant la même hostilité de principe à l'Occident. La charité des notables du début du siècle se trouvait de fait délogée, tant par les politiques de subventions que par les «stratégies de bienfaisance » des mouvements religieux.

La mainmise des mouvements religieux sur la charité continuera dans les années soixante-dix. Les imaginaires avaient certes changé : la pensée libérale légitimait de nouveau la possession de richesses, alors que le renforcement des valeurs islamiques réhabilitait la charité comme pratique signifiante. Mais cela n'allait pas suffire, dans un premier temps, à inciter les grandes familles industrielles et commerciales affaiblies à investir dans des opérations de charité. Ces familles s'avéraient plutôt rivées à un objectif d'accumulation que mues que par des considérations de prestige ou par un sentiment de responsabilité sociale.

Dans l'esprit des militants "religieux", en dehors de ses fonctions utilitaires (encadrement des masses, instrument de mobilisation), la charité constituait une pratique distinctive où l'acte de foi se vivait aussi comme affirmation d'une double altérité : par rapport à un gouvernement corrompu et égoïste, devenu étranger aux valeurs fondatrices qu'il revendique; par rapport à un Occident honni et conçu comme propagandiste d'une culture de la perdition, de l'individualisme, etc. 
12 Attestant de leur volonté de retourner aux principes authentiques, la charité des militants de la réislamisation affirme symboliquement leur opposition à une classe dominante rétentrice tant au niveau du pouvoir que des richesses, en disqualifiant celle-ci à partir du vieux discours qui dénonce son caractère occidentalisé. En deux mots, faire acte de générosité, c'est proclamer que les élites, comme l'Occident, professent l'égoïsme. Le régime et l'Occident sont donc rejetés dans le monde disqualifié de la vanité et de l'avarice pour stigmatiser un modèle de société présenté comme régi par des valeurs purement individualistes, par un hédonisme scandaleux, un goût indu du luxe et se contentant de plaisirs immédiats. Une construction polémique $\mathrm{du}$ sens qui souligne une fois de plus que la vision de l'Occident - et pas seulement l'appropriation de ses catégories - est largement tributaire des rapports de force locaux et des stratégies de délégitimitation des élites au pouvoir. Si l'Occident est conspué, c'est peut-être d'abord parce que l'État est perçu comme lui ressemblant.

13 L'argumentation des militants religieux était assurément fondée dans les années soixante-dix, si l'on en croit les itinéraires d'accumulation des élites économiques: confiscation des rentes, privatisation des ressources de l'État, utilisation des positions publiques à des fins d'enrichissement privé, collusion avec les capitaux étrangers ou les hauts dignitaires du régime, étalage de la richesse nouvellement acquise dans les lieux de l'extraversion (hôtels cinq étoiles, bars, clubs de l'ancienne aristocratie...). Autant d'éléments qui venaient renforcer l'usage des valeurs islamiques comme argument formalisant le rejet des classes possédantes, perçues comme occidentalisées.

\section{La charité des nouveaux entrepreneurs}

14 La situation change dans les années quatre-vingt sous l'effet de deux mouvements : la consolidation d'une nouvelle élite économique, d'abord, et la vitalité du mouvement islamiste, ensuite. Force désormais dominante dans certains quartiers populaires, il devient le principal ennemi du régime.

Dans ce climat de tensions qui dessert leurs intérêts économiques, les entrepreneurs vont réactiver leurs investissements de charité. Leurs exigences d'ordre et de progrès se renforcent, car les affaires demandent l'ordre social, et l'État ne peut manifestement l'assurer à lui seul. Une telle situation les conduit à développer une éthique de la responsabilité sociale d'autant plus prégnante, que, religieusement valorisée, elle servait leurs intérêts de classe. Au total, l'injonction islamique de solidarité, passée dans les mains de la classe d'affaire, pouvait se transformer en une forme de philanthropie du type de celle mise en œuvre, en Occident, par un Rotschild ou par un Rockfeller.

16 Pourtant, une telle évolution n'allait pas de soi. Les liens affirmés de certains grands entrepreneurs avec les Frères musulmans ${ }^{6}$ et les multiples itinéraires de réislamisation dans le champ des affaires rendaient incertaine une telle évolution. Mais une sensibilité à un code culturel ne signifie pas une adhésion à l'idéologie qui s'en saisit; la réaffirmation des catégories islamiques du don chez les entrepreneurs n'est pas de la réislamisation, tout au moins au sens d'un aggiornamento du local ou du religieux. Les hommes d'affaires ne redécouvrent pas la zakât parce qu'ils sont devenus pieux mais parce que celle-ci a des affinités avec le modèle de société qu'ils se sentent désormais en droit de revendiquer : le modèle libéral valorisant la propriété privée et l'entreprise individuelle, modèle dont les préceptes convergent avec l'impératif islamique d'« effort 
personnel » (juhûd dhâtiyya) souvent mis en avant par les hommes d'affaires. La charité réapparait ainsi au point de convergence entre les nécessités sectorielles des entrepreneurs (promouvoir l'entreprise privée), le système de valeurs en cours (l'obligation de solidarité envers les pauvres) et la ligne politique dominante sur la scène internationale (consolider l'État minimum). En d'autres termes, la charité scelle avec bonheur la triple alliance de l'éthique (religieuse), de l'intérêt (de classe) et de l'idéologie mondialement dominante (la promotion du modèle libéral).

On n'attribuera donc pas le retour de la charité dans les rangs des hommes d'affaires à la seule influence qu'exercent sur eux les préceptes de la doctrine islamique. Si la charité refait surface, et si elle apparaît singulièrement acculturée et alignée sur les codes occidentaux, c'est parce qu'elle vient occuper la place vacante que lui a légué la conjoncture contemporaine, locale et globale : retrait de l'État, idéologie néo-libérale, affirmation politique d'une classe d'affaires à la recherche de signes d'excellence, etc. En effet, cette propension toujours plus massive de nouveaux entrepreneurs à faire œuvre de charité ne laisse qu'une alternative: soit il s'agit d'une manifestation supplémentaire de réislamisation, soit la pratique islamique est le médiateur d'un autre message.

Sans évoquer pour cela la "macdonaldisation » de la charité, il est toutefois pertinent de repérer dans ce pied de nez aux affirmations anti-occidentales émanant des cercles religieux, l'indice d'une mutation de fond: les acteurs de la charité ne sont pas nécessairement liés au champ politique et ils ne parlent plus la langue de l'idéologie et de l'identité. Furtivement, sous le discours religieux qu'ils arborent parfois bruyamment, une autre grammaire s'invente, empruntant plus d'une règle aux grammaires exogènes. De fait, il semble que l'on assiste à la déconnection relative des postures identitaristes et de la pratique religieuse (une hypothèse corroborée par l'évolution d'autres conduites religieuses, comme le port du voile ou les normes de pudeur (Haenni, 1996b).

Ainsi les modalités de l'acquittement de la zakât ou de la sadâqa se recomposent en conséquence pour redevenir - et ceci pour la seconde fois de l'histoire moderne des actes de charité - perméables aux influences occidentales. La petite histoire des tables de ramadan (mawâ'id al-rahmân) est suffisamment riche en enseignements pour que l'on s'y arrête un instant. Nous passerons ensuite à des considérations plus générales.

Instaurées par les Fatimides au $\mathrm{X}^{\mathrm{e}}$ et $\mathrm{XI}^{\mathrm{e}}$ siècles, destinées aux pauvres, aux passants et aux voyageurs, les tables de charité deviendront par la suite l'apanage des notables des campagnes. En ville, elles ne sortiront pas de l'enceinte des mosquées. En 1968, la Banque Nasr appartenant à l'État socialiste sera l'instigatrice, au Caire, de cette tradition religieuse. Elle revient dans des mains plus "orthodoxes"-celles des militants religieux - lors de leur expansion dans les années soixante-dix. Religieusement souhaitable, la table de charité, devenait également profitable à ces derniers, leur permettant momentanément de gagner le soutien des ventres vides. Les hommes d'affaires, suivis d'une multitude de propriétaires de magasins et de petits commerçants, allaient, à leur tour, en organiser.

21 À Mohandesseen, un des quartiers cossus du Grand Caire, le Qasr al-malik, un somptueux magasin de meubles est flanqué d'une longue table. En l'espace d'une heure, lors de la rupture du jeûne, s'y côtoient, à peine séparés par la baie vitrée de la boutique, lustres de cristal flamboyants et gallabiyya rapiécées à l'extrême. Le propriétaire? Un homme d'affaires qui a fait fortune dans le Golfe et qui, après sa 
rencontre avec un cheikh d'Arabie Saoudite, s'est résolu à reconvertir une partie de ses investissements en œuvres pieuses. Ismâ'îl Sidqî, l'ancien premier ministre, voit dans ces conduites « le symbole de la coopération en faveur des pauvres et des nécessiteux ». $\mathrm{Et}$, bien des attablés semblent partager son point de vue.

Mais, par delà les louanges, les fausses notes n'ont pas tardé à résonner : la table de charité a désormais ses adversaires, en la personne... des hommes de religion et des islamistes, conscients que la tradition qu'ils avaient contribué à instaurer dans les années soixante-dix est en passe de leur échapper. C'est en 1996 que la croisade contre les mawầid al-rahmân connaîtra son apogée: "Une partie de cet argent est illicite (harâm) et ceux qui l'utilisent sont des professionnels de la mendicité ", écrivait le bihebdomadaire de sensibilité islamiste al-Sha'b, rejetant dos-à-dos tributaires et prestataires de ce qu'il considère comme une charité pervertie. À quoi certains cheikhs devaient rétorquer : «Personne ne peut juger les intentions des donateurs. Quelles que soient ses intentions, celui qui fait la charité sera récompensé... fût-ce malgré lui»; «L'intention des donateurs doit être orientée vers Dieu seulement, loin de la publicité et de la fierté ».

Les avatars des tables de charité sont anecdotiques quoique exemplaires. Au-delà de leurs aspects iconoclastes, ils se font l'écho de mouvements plus amples et témoignent de la réintégration des pratiques de charité dans le champ de l'hybridation culturelle, une tendance que leur monopolisation par les acteurs religieux avait réussi pour un temps à contenir. La diversité de forme des mawà'id al-rahmân traduit donc la présence de fait, sinon de droit, d'alliances non polémiques entre des catégories éthiques de la morale musulmane et certains schèmes de la « culture occidentale ».

\section{Hybridation et juxtaposition des systèmes de références}

Les investissements charitables de Husâm Badrâwî sont exemplaires de cette tendance : ses liens dans le champ associatif comme son itinéraire éducatif lèvent toute ambiguïté : membre du New Civic Forum (voir infra), de l'American Chamber of Commerce, fondateur de Health Care Quality Association, il est aussi en relation étroite avec US AID et membre de l'association For A Better Future. Un itinéraire qui ne pouvait que l'exposer aux influences occidentales qu'il s'est appropriées de manière volontariste et non polémique: militant en faveur d'un libéralisme ventilé de considérations sociales, il défend l'idée d'un droit à la santé pour tous et relève l'incapacité de l'État à l'assurer. Il crée en 1990 le Middle East Medicare, un réseau d'organisations de santé présent au niveau national :

"C'était un changement dans ma vie que de m'intéresser à la mise à disposition de services, non plus dans la perspective d'un investissement, mais du point de vue de ma responsabilité religieuse. C'est au moment où tout en Égypte s'orientait vers plus de respect pour les forces du marché que j'ai réalisé la force de la zakât al-mâl : si vous n'avez pas de rôle social et de stabilité dans la société, vous ne pouvez pas maximiser vos bénéfices. Et la stabilité dans la société ne se crée pas par le gouvernement. Non, ce sont les gens et les ONG (organisations non gouvermentales) qui la font. Les islamistes ont bien compris que la meilleure manière de recevoir le soutien des gens était de les servir directement par l'éducation et un peu de sécurité sociale, et c'est ce qu'ils leur donnent. Ils ont réussi là où le gouvernement a échoué. J'ai repris cet exemple: les gens réclament tout le temps des soins de 
santé. Le gouvernement paye 10 milliards Łe en soins de santé par an et pourtant les gens souffrent et ne peuvent bénéficier de ces services. C'est pour cela qu'il faut laisser le secteur privé mener, mais un secteur privé intelligent qui sache assurer un environnement stable autour de lui pour lui permettre la prospérité de ses entreprises. Si vous êtes égoïste mais intelligent, si vous avez des racines dans votre pays, vous penserez que la stabilité de l'Égypte est une part de votre propre stabilité et de votre crédibilité devant le monde. Parce que vos propres investissements opèrent dans un système international global [...]. Il y a un rôle pour ceux qui pensent comme nous, qui sont éduqués et qui sont prêts à des compromis, à s'engager pour la stabilité. » (Entretien)

Que fait au fond Husâm Badrâwî, fut-ce simplement sur le terrain du discours? Il mobilise simultanément deux registres de sens : une sensibilité islamique d'abord, un second vocabulaire ensuite, où se dessinent en filigrane toutes les catégories du «versant solidaire » de l'économie néo-libérale : «les activités humanitaires sont une manière différente d'exprimer la sadaqa. La sadaqa est religieuse, l'humanitaire est plus large que ce qui m'est demandé par ma religion. Au-delà du minimum imposé par la zakât, il y a une plus-value qui est mon action humanitaire». On ne peut manquer de rapporter ces propos à ce que préconisait Tahtâwî un siècle plus tôt: «les temps modernes ont fait naître d'autres demandes: il faut combattre l'usure, aider les négociants qui font faillite, secourir les travailleurs privés d'emploi. [...] Pour prendre en charge ces nouveaux besoins, la sadaqa doit donc revêtir des formes nouvelles, celles des sociétés de secours mutuel ou de bienfaisance qui viendront compléter les œuvres individuelles de la tradition musulmane » (cité dans Alleaume, à paraître).

Comme pour Rifâ'a al-Tahtâwî, les conceptions occidentales de la charité fournissent un mode de pensée et d'action, mais les vecteurs ne sont plus les mêmes aujourd'hui : si ce sont largement des personnalités proches du monde de l'enseignement qui ont véhiculé les principes charitables de l'économie sociale du $\mathrm{XI}^{\mathrm{Xe}}$ siècle, la pensée humanitaire s'installe, elle, par l'intermédiaire des praticiens, c'est-à-dire, par la nébuleuse des ONG et des institutions gouvernementales internationales qui accompagnent les programmes d'ajustement structurel. Le soutien au secteur privé dispensé par celles-ci, l'accroissement du nombre des partisans locaux du libéralisme, la multiplication des formations à l'étranger, la présence accrue des institutions internationales dans la vie sociale et économique locale, la multiplication des «joint ventures » dans le cadre des projets de développement ne cessent d'étendre le milieu susceptible de subir l'influence de l'idéologie humanitaire. Les entrepreneurs de «l'après-infitâh)» ont placé une part notable de leurs investissements de solidarité dans ce réseau diffus, ajoutant à leurs attitudes ostentatoires des conduites évergétiques et philanthropiques.

Une autre différence entre la charité des entrepreneurs et celle des réformistes du siècle passé se situe dans la position adoptée par ceux-ci vis-à-vis de l'Occident. Pour Tahtâwî, en effet, l'Occident constituait certes une référence mais l'importation de ses catégories restait conditionnée par leur homologation (Ferrié, 1996), en d'autres termes, l'accès à l'Occident se faisait d'abord par la médiation du local parce que conformément à l'orientation des acteurs de la Nahda, l'Occident restait appréhendé à partir d'une position identitariste. Pour Badrâwî, au contraire, la mobilisation des idiomes locaux vise l'ouverture à l'Occident plus qu'elle ne sert de cadre à son accès. En d'autres termes, il n'y a plus simplement encodage des schèmes culturels de l'Occident et médiation par le local, mais accès direct aux catégories exogènes dans une posture qui revendique «le droit de faire du neuf» (Ferrié, 1997) et d'exercer ce droit 
librement. Entre Tahtâwî et Badrâwî, le rapport à l'Occident s'avère ainsi différent et traduit bien les modalités possibles de circulation des objets ou des pratiques occidentales en dehors de leur espace culturel de production. Chez le premier, l'accent est mis d'abord sur l'encodage, alors que chez le second, la valorisation des référentiels locaux demeure présente mais en position subordonnée : le local motive la charité mais ne détermine pas ses formes, il exerce une impulsion sur la pratique plus qu'il ne la dirige.

Les appels du président Moubarak à l'allègement de la dette publique en 1985, ceux de sa femme lors du tremblement de terre en 1992, et l'ampleur de l'événement lui-même furent autant d'incitations politiques à la générosité des hommes d'affaires ${ }^{8}$. En juin 1996, 115 écoles et 400 unités de soins médicaux ont été créées ou modernisées par le "Comité de bienfaisance des hommes d'affaires ». Le soutien aux orphelins mais aussi à des œuvres en faveur de l'éducation ou de l'environnement ou encore l'aide apportée à des projets émanant de petites entreprises, tel est le champ d'action des entrepreneurs. À ceci, il faut ajouter le mécénat: Ahmad al-Maghribî, membre du Conseil d'administration de la société franco-égyptienne Accor établit une connexion supplémentaire avec les conceptions occidentales du bienfait lorsqu'il fit don, en novembre 1996, de 100000 £e pour soutenir la rénovation d'antiquités pharaoniques à Louxor. Tahtâwî semble ainsi avoir trouvé une postérité paradoxale chez les nouveaux entrepreneurs : ils partagent son orientation libérale qui donne la priorité aux objectifs économiques et à l'action privée. Ils s'accordent sur la nécessité de se préoccuper des foyers de tension que représentent les classes populaires et donc de gérer les tensions sociales en recourant aux ONG. Or, une telle ambition suppose que ces organisations soient socialement légitimes et économiquement crédibles : les références faites au cadre religieux serviront à les légitimer tandis que le recours aux institutions internationales procurera les ressources logistiques et financières. D'où, encore une fois, le brassage des normes dans le discours des promoteurs du «Comité de bienfaisance des hommes d'affaires ", évoquant tour à tour "l'importance de la zakât dans le développement de l'éducation » et « le rôle social du capital ». Les révérences faites à la zakât ne sont donc pas purement et simplement imputables à la réislamisation : l'esprit de zakât s'inscrit aujourd'hui dans "l'effort privé en faveur du développement et de la croissance», comme le répétera un de mes interlocuteurs, ajoutant que verser des fonds à une ONG lui permettait « d'être tranquille avec Dieu ».

Ces illustrations invitent à deux constats : premièrement, les transformations de la charité ne peuvent être pensées en dehors de la présence de l'Égypte sur la scène mondiale, notamment par le maillage de plus en plus serré entre les institutions de bienfaisance des élites locales et les réseaux de l'aide humanitaire internationale; deuxièmement, les mécanismes d'appropriation positive de l'Occident opèrent aussi dans le champ religieux. Il reste que l'univers dans lequel les entrepreneurs ont choisi de s'inscrire n'est pas moralement neutre et ses principes ne sont pas forcément en accord avec l'éthique musulmane. Et, assurément, il est pour eux de plus en plus difficile de se situer en dehors du système de référence des organisations humanitaires : l'universel abstrait et désincarné. Cet universel n'est pas l'universel de la zakât, laquelle a ainsi paradoxalement favorisé la réception de références qui ne sont pas les siennes. Or, de fait, cette conception s'accommode difficilement de l'universel islamique qui est porteur d'une dimension ethnique prépondérante. Les entrepreneurs ont donc dû gérer la rencontre de ces conceptions contradictoires. Ce qui, par 
l'adhésion des entrepreneurs à des vérités contraires allait se faire, non sur le mode du brassage, mais sur celui de la coexistence.

En effet, plutôt que de tenter de tisser des convergences incertaines entre des cadres de pensée fondés sur des prémisses différentes, il vont simplement « dédoubler » leurs pratiques de charité et on les verra, d'un côté, se mobiliser sur une ligne particulariste et, de l'autre, souscrire aux préceptes de leurs partenaires occidentaux, sans qu'ils se posent la question de la compatibilité des "programmes de vérité » qui les régissent (Veyne, 1983): ici universalisme communautaire et universalisme individualiste se côtoient, nonobstant leurs principes incompatibles. Ils évitent donc tout dilemme par l'occultation du différend et non par un effort de synthèse. On le voit, les rapports entre le local et le global ne sont pas toujours régis par une relation dynamique de syncrétisme aboutissant à leur recomposition mutuelle (Haenni, 1996a). Ceci constitue un cas de figure mais ce n'est pas le seul : ces rapports peuvent aussi se régler par leur simple occultation. Et c'est peut-être là la figure majoritaire : la zakât comme valeur éthique motive le travail humanitaire mais, en tant que norme, elle continue à coexister de manière hétérogène et non conflictuelle à côté du monde des ONG transnationales. Cela est possible parce que la référence n'est pas rapportée à ses principes fondamentaux. Husâm Badrâwî n'y échappe pas. D'un côté, on le voit tisser son ancrage social par le biais d'un engagement local assidu, subventionnant la mosquée où il a l'habitude de se rendre, patronnant une table de charité durant le mois de ramadan pour "prendre soin des membres de la famille et des proches du quartier ». D'un autre, on le retrouve aux bals de charité du Lion's Club, aux réceptions de l'US AID. Il vit en effet entre deux « mondes ", deux systèmes de références porteurs d'idéaux de perfection différents qui, en l'occurrence, ne se croisent pas.

\section{Conclusion}

31 Qu'en est-il donc de la posture réformiste vis-à-vis de l'Occident? On la retrouve du côté de l'intelligentsia religieuse progouvernementale. Le discours savant des doctes aura tôt fait de proposer une argumentation utilitaire et fondamentalement consensuelle de la zakât reprenant les recommandations de Tahtâwî. Il s'agit de nouveau, un siècle après lui, de mobiliser les forces religieuses pour amortir les coûts de l'adoption d'une économie basée sur les lois du marché. En ce sens, la zakât relève bien de la politique. L'Occident cessait d'être, dans leur vision, la cible conspuée des militants islamistes pour devenir une source d'inspiration dans la négociation du virage néo-libéral amorcé par le gouvernement égyptien. Fort logiquement, le discours des doctes allait rejouer la carte de l'hybridation et brasser sans polémique les catégories locales et exogènes. Quand le Centre d'études stratégiques et politiques al-Ahrâm, intitule un des ateliers de son séminaire "les versants économiques, sociaux et humanitaires de la zakât ", quand dans ce même atelier, l'un des intervenants déclare que «dans notre position matérielle actuelle, nous trouvons dans la zakât et ses obligations, l'issue naturelle aux problèmes nés du développement économique contemporain ", on voit les conceptions islamiques réactivées dans un dessein pour le moins éloigné, tant du cadre éthique et moral traditionnel que du projet islamique : gérer les effets pervers du modèle libéral d'inspiration occidentale. Lorsque le même participant affirme ensuite que la zakât est un mécanisme susceptible de contrecarrer l'évasion fiscale, il est clair que la norme locale (i.e., la zakât) vient - en dehors même de 
toute stratégie de légitimation - sanctionner positivement la norme exogène (i.e., les schèmes économiques du modèle néo-libéral de société).

Cet objet apparemment si traditionnel et si endogène que constitue la charité a été en réalité constamment façonné par les problématiques dominantes du temps mondial en matière de solidarité sociale. Les nouveaux entrepreneurs font donc de la bienfaisance en se référant à l'éthique musulmane. Pour autant, ce ne sont pas des puristes : leur démarche n'est pas conduite par le désir de restaurer un idéal perdu mais par la recherche de la performance et de l'excellence. Or, l'Occident est riche de ressources que mettent à disposition les ONG. L'attitude charitable des hommes d'affaires - l'esprit de zakât-, allait donc prendre la forme de l'action humanitaire et quitter de plus en plus les institutions religieuses traditionnelles. Si un tel mouvement s'est avéré possible, c'est parce que leur vision de l'Occident s'est en quelque sorte délocalisée, contrairement à la perspective dans laquelle s'inscrivaient Tahtâwî et le mouvement réformiste. S'ils s'accordent sur une visée - l'accroissement du bien-être -, le cadre idéologique dans lequel cette visée est inscrite s'est modifié : le XIX ${ }^{e}$ siècle avait la Nahda comme idéologie dominante et son principe même, la renaissance, déterminait le rapport possible à l'autre : celui-ci était surdéterminé par l'exigence de régénération de la culture locale. Or, ce principe n'a plus la même force et son affaiblissement facilite, de fait, l'accès à l'Occident dès lors qu'il n'est plus seulement perçu en termes identitaires mais, bien plutôt, dans une perspective pragmatique. L'esprit de zakât a donc favorisé le mouvement d'ouverture des conduites de don des entrepreneurs égyptiens sur les canons occidentaux de la charité. La logique de l'ouverture est peutêtre en train de prendre le pas sur la logique réformiste de l'encodage.

\section{BIBLIOGRAPHIE}

ALLEAUME G. :

- 1995, « La réforme sociale vue par les dirigeants de l'industrie agricole », dans ROUSSILLON A., Entre réforme sociale et mouvement national, identité et modernisation en Égypte, 1882-1962, Le Caire, Cedej.

- à paraître, «L'économie politique saint-simonienne et les élites techniques de l'Égypte », dans Etudes saint-simoniennes, Lyon, Presses universitaires de Lyon.

FERRIÉ J.-N. :

- 1996, «L'appartenance des objets. Problèmes d'anthropologie de la culture et de l'identité », Égypte-Monde arabe, $\mathrm{n}^{\circ} 25$.

- 1997, « Le bébé et l'eau du bain : droit de faire du neuf, espace public délocalisé et démocratie de marché », dans LAÏDIZ., Le Temps mondial, Bruxelles, éd. Complexe.

HAENNI P. :

- 1996a, «Libéralisation et libéralités des entrepreneurs en Égypte. Éléments pour une sociologie de la dépense », Égypte-Monde arabe, $n^{\circ} 25$. 
- 1996b, « Les logiques sociales de la réislamisation », Égypte-Monde Arabe, n 26.

HANNA N., 1997, Making big money in 1600. Biography of Ismail Abu Taqiyya, Shahbandar al-tuggar, Cairo, American University of Cairo.

HAYNES D., 1987, «From Tribute to Philanthropy : the Politics of Gift Giving in a Western Indian City », The Journal of Asian Studies, vol. 46, $\mathrm{n}^{\circ} 2$.

ILBERT R., 1995 « Modèles et vecteurs de la réforme. Le libéralisme des notables ", dans ROUSSILLON A., Entre réforme sociale et mouvement national, identité et modernisation en Égypte, 1882-1962, Le Caire, Cedej.

JAFFRELOT C, 1994, « CEuvres pies », dans BAYART J.-F., La Réinvention du capitalisme, Paris, Khartala.

ROUSSILLON A., 1995, «Introduction. La modernité disputée : Réforme sociale et politique en Égypte ", dans ROUSSILLON A., Entre réforme sociale et mouvement national, identité et modernisation en Égypte, 1882-1962, Le Caire, Cedej.

'UMAR M., 1902, Hâdir al-misriyyin aw sirr ta'akhkhurihim (L'État présent des Égyptiens ou le secret de leur arriération), Le Caire, Al-Muqtataf.

VEYNE P. :

-1976, Le pain et le cirque. Sociologie d'un pluralisme politique, Paris, Seuil.

-1983, Les Grecs ont-ils cru à leurs mythes? Paris, Seuil.

\section{NOTES}

1. La catégorie la plus formelle est la zakât, l'aumône légale faisant partie des cinq piliers de l'islam, c'est-à-dire des obligations imposées au croyant. Institutionnellement, elle donnera lieu aux awqâf (biens de mainmorte). D'autres notions comme la sadâqa, la takâful renvoient de façon plus lâche à l'injonction religieuse de «solidarité envers les pauvres". Nous utiliserons indifféremment l'une et l'autre de ces notions. De même, nous ne ferons pas de distinction entre « charité » et « bienfaisance ».

2. Le mouvement islamiste notamment a contribué à transformer la charité en une pratique contestataire, inscrite dans un projet politique et idéologique d'ordre identitaire, qui vise, tout à la fois, à la réislamisation de la société et au retour à une origine mythique épurée de toute influence occidentale.

3. Les quelques propositions ici sont sans aucun doute simplificatrices. Nous totalisons assurément une époque riche en diversité. Toutefois, cet idéal-type brossé en quelques lignes a pour intérêt de décrire un ensemble de pratiques contre lesquelles les pionniers de la réforme des conceptions et des pratiques de la charité vont se positionner par la suite. Leur programme avait pourtant des antécédants. Les grands commerçants de la période ottomane notamment situeront leurs investissements de prestige et de charité bien au-delà du cadre étriqué des solidarités locales: "They emphasized their status by their large scale projects which were an announcement of their new position in society. Not only commercial buildings, religious and charitable buildings, but whole quarters now bore the names of prominent merchants. "(Hanna N., 1997, p. 31)

4. M. 'Umar la définit comme « l'appartenance à une maison commune, dans les liens de parenté et le partage d'une même filiation. Puis elle s'étend des membres d'une même maison au voisin, puis au voisin du voisin [...] jusqu'au quarantième voisin » ('Umar, 1902, p. 10).

5. Le vocabulaire même de la Lajnat i'ânat fuqarâ'al-muslimîn al-wataniyyîn (Ligue des pauvres musulmans patriotes) créée en 1892 donne une idée du poids persistant des catégories 
religieuses. Il en va de même pour la Société de la foi pour l'entraide de ceux qui œuvrent conformément au livre Saint et à la tradition muhammadienne, fondée en 1912 par le réformiste Muhammad 'Abduh.

6. Citons, par exemple, l'entrepreneur Osman Ahmed Osman dont les sympathies pour les Frères musulmans sont connues. Par ailleurs, on se souvient de onze hommes d'affaires dont les noms figuraient sur les listes islamistes lors des élections législatives de 1996.

7. Il a fait ses études post-universitaires à Wayne State University Michigan où il s'est spécialisé dans la contraception. Il a ensuite travaillé à l'hôpital universitaire de Chicago pendant un an et demi. Il a suivi un stage de formation complémentaire à Boston University, où il sera maître de conférence d'abord, et professeur par la suite, avant de revenir au Caire en 1993.

8. L'opération Hubb Misr (« Amour de l'Égypte ») fut lancée par le président Moubarak en 1985 pressé alors par la communauté internationale de régler les arriérés de payement des emprunts du gouvernement égyptien. À cette occasion, appelant à la conscience civique des citoyens, le président Moubarak les exhorta à contribuer de leurs deniers afin d'alléger le fardeau de la dette publique. On se souvient notamment de la rencontre au sommet entre les deux équipes-phare du Championnat égyptien al-Ahlî et al-Zamalek, destinée à promouvoir l'appel du président. À cette occasion, un groupe d'hommes d'affaires descendit sur la pelouse du stade du Caire, distribuant prix et éloges à ceux qui eurent le geste large en faveur de la nation. Suzanne Moubarak ne demeura pas en reste non plus, puisque sept ans plus tard, lors du tremblement de terre de 1992, elle appela à la solidarité du peuple égyptien en faveur des victimes. Deux comptes furent ouverts au nom du Croissant Rouge où les dons pouvaient être adressés. Les comptes, sous la surveillance étroite de la première dame d'Égypte, étaient notamment destinés à contrer les solidarités qui s'organisaient sous le contrôle des différentes ailes du mouvement islamiste.

\section{INDEX}

Mots-clés : islamisme, Occident, zakât, charité, éthique

\section{AUTEUR \\ PATRICK HAENNI}

Cedej 